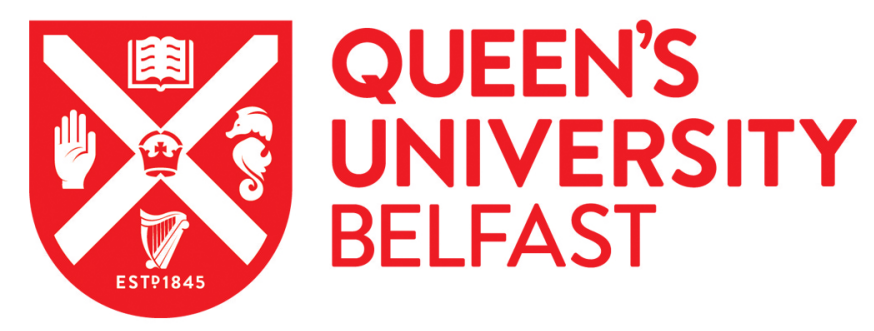

\title{
Tolerance aware product development using an enriched hybrid digital mock up
}

Friel, I., Butterfield, J., Robinson, T. T., \& Marzano, A. (2020). Tolerance aware product development using an enriched hybrid digital mock up. CIRP Journal of Manufacturing Science and Technology, 30, 118-130.

https://doi.org/10.1016/j.cirpj.2020.04.009

Published in:

CIRP Journal of Manufacturing Science and Technology

Document Version:

Peer reviewed version

Queen's University Belfast - Research Portal:

Link to publication record in Queen's University Belfast Research Portal

Publisher rights

(c) 2020 Elsevier B. V.

This manuscript is distributed under a Creative Commons Attribution-NonCommercial-NoDerivs License

(https://creativecommons.org/licenses/by-nc-nd/4.0/), which permits distribution and reproduction for non-commercial purposes, provided the author and source are cited.

\section{General rights}

Copyright for the publications made accessible via the Queen's University Belfast Research Portal is retained by the author(s) and / or other copyright owners and it is a condition of accessing these publications that users recognise and abide by the legal requirements associated with these rights.

Take down policy

The Research Portal is Queen's institutional repository that provides access to Queen's research output. Every effort has been made to ensure that content in the Research Portal does not infringe any person's rights, or applicable UK laws. If you discover content in the Research Portal that you believe breaches copyright or violates any law, please contact openaccess@qub.ac.uk. 


\title{
Tolerance Aware Product Development Using an Enriched Hybrid Digital Mock Up
}

\author{
Friel, Imelda ${ }^{1}$ Butterfield, J. ${ }^{2}$ Robinson, Trevor Thomas ${ }^{2}$ \\ Queen's University Belfast, Belfast, County Antrim, BT7 1NN, Northern Ireland \\ and \\ Marzano, Adelaide ${ }^{3}$ \\ University of the West of Scotland, Paisley, PA1 2BE, Scotland
}

\begin{abstract}
Current practice in three-dimensional (3D) part construction and product assembly within a digital mock-up (DMU) is based on the nominal sizing of geometry. This approach does not reflect real part dimensions when they are manufactured or the assembly conditions as they come together during the final build. The value of virtual assembly planning (VAP) using clash and gap detection is therefore limited to modelling error identification within the computer-aided design (CAD) domain for nominal conditions only. VAP could become a significantly more powerful tool if the 3D representation of components within the DMU were more representative of 'as manufactured' dimensions. This paper presents the evolution and validation of an enriched DMU (EDMU) introducing the concept of the hybrid assembly which represents components in sizes which can range between upper and lower dimensional limits in addition to the nominally defined form. The resulting capability has been exploited to enable a designer to perform tolerance analysis in the CAD domain in advance of manufacturing planning, allowing tolerance consideration earlier in the design process as the DMU evolves. This has the potential to reduce the time, effort and computational load associated with the application of statistical tolerance analysis by methods engineers later in the product development cycle when the costs associated with change are higher.
\end{abstract}

Keywords: Computer-aided design; Variational geometry; Tolerance

\footnotetext{
${ }^{1}$ Postgraduate Research Student, School of Mechanical and Aerospace Engineering. Student Member

${ }^{2}$ Senior Lecturer, School of Mechanical \& Aerospace Engineering.

${ }^{3}$ Senior Lecturer, School of Engineering \& Computing.
} 


\section{Introduction}

DRODUCT development, in any engineering or industrial setting, is a multi-stage, multi-disciplinary process. It spans conceptual design through to the final release of drawings and models for part manufacture, product assembly, quality checks and distribution to the customer. It is acknowledged that the continuing advances in manufacturing technologies must be better supported throughout the design process [1-3] in order to improve the quality and chronology of manufacturing planning and execution. Original equipment manufacturers (OEMs) require ways to improve their product development process in order to satisfy customer demands for high quality products in shorter lead times [3-5]. Integrating predictive methods for manufacturing planning within the design process has the potential to satisfy this requirement as digital manufacturing methods can be used for process validation as well as the capture of production critical knowledge within the digital domain.

It is during the design phase that a DMU is created using CAD software. The product begins as a basic conceptual model to which greater levels of detail are added as the DMU evolves into a virtual product reference [6]. This is used within product lifecycle management (PLM) systems for the purpose of manufacturing planning. However, product geometry within the DMU is only ever modelled in a nominal form which limits the potential of digital manufacturing methods for the accurate simulation of production conditions in terms of realistic component dimensions and fits during assembly.

Dimensional tolerances are an important governing factor in the manufacture, assembly and ultimate function of any given product in any engineering discipline. They effectively make allowances for the range of possible variation between the nominal form and the real product dimensions. Although statistical methods can be used to inform tolerance analysis and allocation, this is typically executed using computer-aided tolerancing (CAT) software when a design and its associated DMU have reached maturity. There is currently little or no provision for the consideration of 'as manufactured' part geometries during the earlier phases of the design cycle outside the application of the tacit knowledge of the designer based on experience. Design interventions to eliminate issues due to tolerance stack-up do not typically take place until a point in the development timeline where time and cost impact around design changes are significant. The opportunity to consider the impact of dimensional variability as the DMU evolves will improve the designer's understanding of their effect on the assembly, enabling the establishment of a tolerancing scheme as individual part models and sub assembly schemes become available. This has the potential to alleviate the work load 
of methods engineers using CAT software during the latter stages of product development to deliver the time and cost savings made possible through better use of predictive methods in manufacturing planning.

The aim of this work is to enable better informed design for manufacture from a dimensional tolerancing perspective, by providing embedded modelling functions within the 3D CAD environment through the co-existence of nominal and realistically sized component forms. This work builds on principles developed by Wan Din et al. [7] who used simple geometric forms to define tolerances on key control characteristics (KCCs) based on the tolerance limits of the key product characteristics (KPCs) based on a CAD parameter sensitivity analysis. KCCs are the manufacturing dimensions that contribute to the overall assembly dimension and require a tolerance limit. The KPCs are the assembly dimensions which are toleranced to ensure effective assembly function. For this work, Wan Din's Wheel Mount assembly exemplar [7] with its defined KCCs and KPCs provided the basis for the creation of a 'Toleranced Hybrid' assembly which includes the functionality to create versions of each component sized between the maximum and least material conditions. It was used to perform 'worst-case' dimensional analysis as a means to verify the tolerance scheme of the model, by validating the original tolerance limits of the KPCs. Equally, if these KPC limits were unknown as is the case when a designer is considering tolerances, this method shows the configurations for which maximum deviation of the nominal KPC is seen, based on the given KCC tolerances, in order to inform and direct the definition of its tolerance limits. Having been developed and then applied to a simple Block Assembly, the methodology was then extended to the key functional components within an Internal Combustion Engine and the structural elements within a Stiffened Aircraft Panel. The work shows how the ability to represent alternative forms of the nominally defined product within the CAD domain can aid the designer in dimensional tolerance analysis by making more realistically sized part forms available within the DMU.

\section{II.Literature Review}

\section{A. DMU in product development}

The DMU is a design tool that allows the virtual assembly of a product to serve as a visual reference for all of the engineering disciplines associated with the product's development. Airbus* refer to the DMU as 'a "Master"

\footnotetext{
${ }^{*}$ http://www.airbus.com/innovation/proven-concepts/in-design/digital-mock-up/
} 
reference' in their A350 XWB program. From its beginning at the concept design phase to its use throughout the entire lifecycle of the aircraft, the DMU ensures that up to date information is accessible to all teams and individuals who are working concurrently on the program. The DMU has been defined in broader literature as a digital 3D representation of a product, its assemblies, structure and attributes - a 'virtual product reference', $[6,8,9]$. In the late 1980s, the Boeing Company pioneered the DMU as the major design tool during the development of their B777 aircraft [10]. A reduction of $\$ 22.5$ million in rework and redesign alone was cited by the company as a direct result of the move from the expensive physical prototype to the digital prototype. Since then, this digital prototype has been synonymous with product development, becoming the 'center of communication' between all project members company-wide [4].

The DMU itself consists of CAD components modelled with nominal dimensions and relatively positioned in 3D space. These components are managed alongside technical information in a product data management (PDM) system. This allows ubiquitous access to the models which support multiple processes across the disciplines associated with the project (structures, aerodynamics, methods engineering, production planning etc.). However, insufficient information within these models still means that significant model preparation is required if they are to be used for anything other than a nominal dimensional reference e.g. preparation of geometry for meshing in Finite Element Analysis (FEA) simulations. The DMU is a powerful 'verification' tool [11] for ensuring product functionality, assembly and maintenance process design, kinematic simulations and data visualization throughout the product lifecycle [12] but significant opportunities remain to improve their utility.

Much research has been done to improve the DMU for downstream processes, for use in simulations [13-15] and to support manufacturing at systems level [16]. Nolan et al. [14] present the concept of Simulation Intent which employs model preparation techniques to ensure robust links between design and analysis models, specifically for FEA simulations. The concept employs three technologies, in particular, 'Equivalencing' between different models of the same cells, to maintain the links between original geometry and their FEA specific representations. Mas et al. [16] presents the "Industrial Digital Mock-Up" (IDMU) as a common platform accessible by all stakeholders in the product lifecycle to achieve optimal design. It was created as a virtual assembly line, through a customized environment in Delmia V5 Manufacturing Hub and a process planning tool, from which shop-floor documentation was automatically generated supporting systems level operations. 
Considerable research effort is focused on improving the DMU for simulations through model preparation activities, primarily for FE simulations [13-15], however, from a manufacturing perspective, a more pressing limitation of the DMU is that it does not represent the 'as manufactured' product [17]. This can be attributed to the fact that CAD systems, the originators of the DMU models, are not 'fit for purpose' when it comes to representing design geometry in the modern manufacturing era [18] and $21^{\text {st }}$ century simulation based tools.

\section{B. Tolerance design in product development}

Tolerance design is an important part of product development. The process involves the specification, allocation and analysis of tolerances that will control variation, linking design with manufacturing to ensure product functionality and customer satisfaction. The tolerance design process requires significant skill [18] and thus is reserved for experts in the field to perform.

Currently, tolerances are represented in CAD systems as two-dimensional (2D) references on drawings, or as 3D annotations on assemblies. The primary role of tolerancing within CAD systems is to serve as a carrier of design intent, for reference during production or inspection of parts or as visualization in design reviews. The lack of practical application of tolerances within CAD limits the use of digital model geometry for accurate manufacturing simulations. With industry's outlook of using CAD assemblies earlier in design for analysis, optimization and simulations [14, 19] it is important to bridge the gap between tolerance modelling in CAD systems and the lack of manufacturing specific information in a DMU.

The research area for tolerance design is vast and remains a hot topic as the manufacturing industry continues to expand into new techniques and processes whilst retaining or improving quality and delivery lead times. Motivation for research is driven by the fact that deviations in individual parts has a cumulative effect on the function and quality of the final product, but due to the limitations of tolerance representation in CAD software, the user can be unaware of these effects until relatively late in the design cycle. Franciosa et al. [20] present a method to perform statistical variation analysis on an assembly having created variational geometry using a morphing mesh approach. This has been demonstrated as an advantage over commercial software capabilities. Xu and Keyser [21] use a LPGUM (Linear Parametric Geometric Uncertainty Model) to create a tolerance zone on a 'target primitive' or feature of interest, knowing the variation of the other primitives. This method is then used to optimize the dimension of a $2 \mathrm{D}$ part according to a selected 'target primitive' tolerance input. Both of these applications extract the CAD geometry and 
perform the analysis outside of the CAD system for user interpretation. A method presented by Ghali et al. [22] performs tolerance allocation enriched by a FMECA (Failure Mode, Effect and Critical Analysis) tool. The tool is used to compute manufacturing difficulty coefficient for feature dimensions (MO) based on the identification of machined (MT) features of a CAD model. This difficulty coefficient is then integrated in tolerance allocation calculations on a rotor key base assembly example resulting in relaxed or tighter tolerances on specific dimensions as well as reduced assembly cost.

Geis et al. [23] directly represent tolerances on the 3D model within the CAD system by transforming ISO standard tolerances defined on the model to vectorial tolerances. Simulation of an assembly within a measuring machine were carried out to demonstrate how the accumulation of tolerances reduced the measurement accuracy of the machine. Only the results of the analysis can be seen on the CAD model as highlighted faces as the method represents tolerances as embedded properties of the surfaces, not as variational geometry. Chan et al. [24] modelled realistic geometries directly in the CAD system using a combination of nominal CAD geometry, variational geometry using tolerance information and fractal geometry of surface. The assembly of a guide rail and sliding table was used to assess the quality based on mating and accuracy of final assembly. Liu et al. [25] focused on the representation of hole variations in particular, developing an algorithm to generate variational geometry based on a degrees-of-freedom (DOF) approach. The algorithm is used to present the hole variation of a mechanical bracket. This method has only been used on a part and not on an assembly. Geis et al. translate predefined tolerances on the CAD model in to vectorial tolerances, meaning the tolerance knowledge is embedded within the model as annotations. Chan et al. [24] use FEA software to incorporate the fractal geometry when a more defined tolerance issue has been identified. Tlija et al. [26] provide a novel model for non-rigid assembly tolerancing by FEA by representing 'realisitic parts' within the assembly through an approach [27 Korbi] that extracts the nominal parts, uses FEA to get deviations of parts under stress loads and using this point cloud data, creates an STL file of the deformed model in CATIA V5. This model is then used to validate tolerances predefined on the model. This use of both embedded tolerance knowledge and third party applications (FEA) for tolerance representation adds a layer of complexity to the process that the designer is not necessarily equipped to use or interpret. There is, therefore, an opportunity to develop embedded functionality within the CAD model itself to facilitate more tolerance aware product development through robust creation and management variational geometry. 
Other work looked at improving the representation and integration of tolerances between the user, the CAD system and other Computer Aided software. Qin et al. [28] presented an algorithm consisting of a meta-model of geometric tolerance zones (GTZ) and a set of generation rules which automatically generated the GTZ associated with the specified tolerance. The algorithm was successfully demonstrated on a gear reducer part; however, this work concerned itself with automating the tolerance design process and did not examine the potential of these tolerance zones in assembly simulations within CAD systems. Litwa et al. [29] created a tolerance analysis model to support the dynamic product development process that caused changes to the tolerance distribution of a feature. The 'skeleton' model aimed to improve the integration issues between CAD and CAT software, but did not extend its application to the simulation of assembly conditions.

It is evident from literature that there is an opportunity to enhance commercial CAD functionality, not only to improve the designer's approach to the tolerance design / allocation process but to allow for more accurate assembly simulations. This literature review has shown how consideration of variations in a $3 \mathrm{D}$ model in a more practical manner has the potential to improve assembly simulations and the user's understanding of the effects of dimensional variation on tolerance stack-up and assembly function. This is not currently facilitated by the current CAD functionality for nominal part modeling, rather through the use of third party CAT software or the application of tacit knowledge gained through experience. The functions required to execute an improved, more integrated approach to tolerance allocation with provision for the co-existence of nominal and variational part versions, do not exist and are not currently used for manufacturing planning. By enriching design with a method for considering tolerances using realistic part forms, the DMU has the potential to become an important manufacturing analysis tool. The approach advocated here will create an enhanced DMU to be used for investigations into the effects of dimensional variation/tolerance stack-up from multiple sources e.g. manufacturing process /alternative assembly sequences. Intrinsically modelling and linking variational geometry within the CAD domain will mean that the practice becomes ubiquitous throughout product development as information and methods are available to perform synchronous design and tolerance analysis in a timely and efficient manner. 


\section{III.Method}

\section{A. Background}

A tolerance allocation procedure has been developed by Wan Din et al. [7] and demonstrated on a simple block assembly model shown in Fig. 1. Using the DMU and defined KCC and KPC parameters, Fig. 2, a sensitivity analysis method was used to define the tolerance stack-up equations for the DMU, relating the KPC dimensions to their contributing KCC dimensions, Eq. 1(a) and 1(b).

Using these equations, the defined tolerance limits on the KPC dimensions are allocated to the KCC dimensions, as shown in Table 1. This method resulted in the numerical values for the KCC tolerance limits, i.e. upper and lower tolerances on their nominal dimensions. The paper suggests tolerance analysis is possible using the CAD parameter sensitivities to control the required dimensional changes in the KCCs but the author did not demonstrate this. The knowledge that these calculations provide is not captured or contained within the environment from which it was gained. CAD supports the definition of tolerances as annotations on drawings or models but design continues using nominal forms, without the understanding of how these variations in dimensions affect assembly fits / relationships.

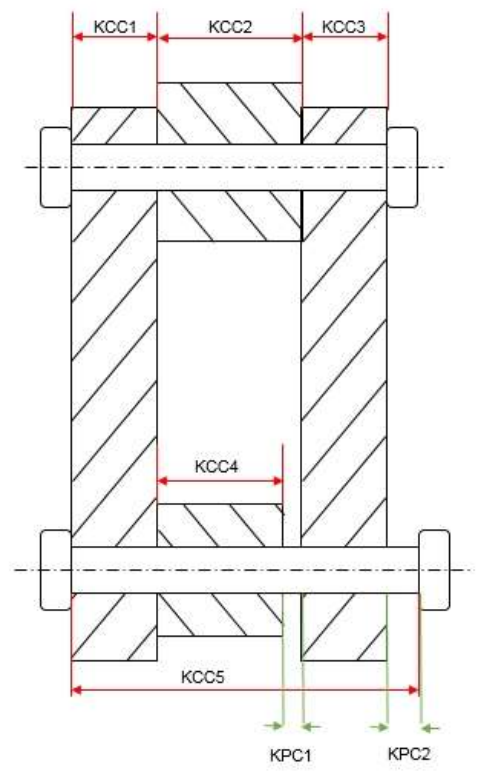

Fig. 2 Key characteristic definitions on the

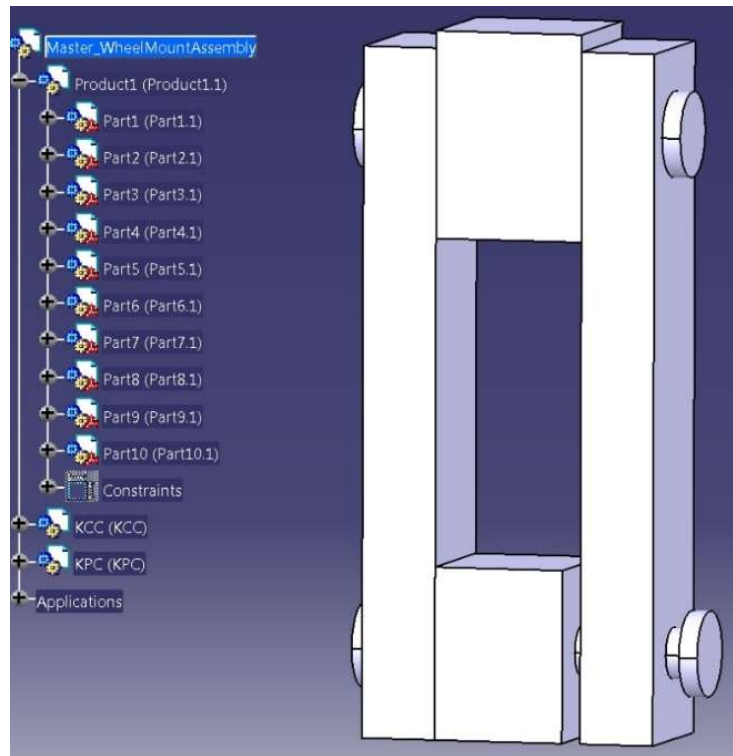

Fig. 1 Simple Block Assembly

\section{Simple Block Assembly}




$$
\begin{gathered}
K P C_{1}=K C C_{2}-K C C_{4} \\
K P C_{2}=-K C C_{1}-K C C_{2}-K C C_{3}+K C C_{5}
\end{gathered}
$$

\section{Table 1 Nominal dimensions and tolerance limits of KC dimensions}

\begin{tabular}{lccc}
\hline \hline & Nominal $(\mathrm{mm})$ & Upper limit $(\mathrm{mm})$ & Lower limit $(\mathrm{mm})$ \\
\hline KCC1 & 20 & +0.1 & -0.1 \\
KCC2 & 40 & +0.4 & -0.4 \\
KCC3 & 20 & +0.1 & -0.1 \\
KCC4 & 35 & +0.3 & -0.3 \\
KCC5 & 85 & +0.1 & -0.1 \\
KPC1 & 5 & +0.7 & -0.7 \\
KPC2 & 5 & +0.7 & -0.7 \\
\hline \hline
\end{tabular}

This method establishes a tolerance allocation on the KCCs based on the availability of the KPC tolerance. The numerical results are not captured or stored within the CAD environment as tangible, modelled features. Wan Din's functionality, therefore, remains numerical and does not result in embedded knowledge within the product development software. Wan Din's work has contributed to the development of the methodology in this paper which enables the tangible representation of the parts in their variational form based on the results obtained from the tolerance allocation method. This in turn enables CAD based learning and understanding of tolerance stack-ups based on 3D geometry which does not require predefined statistical information or historical knowledge on the part of the user.

\section{B. Tolerance Hybrid Assembly}

The simple block assembly with the validated tolerance scheme was used to develop a method to create variational geometries of parts within the assembly, to aid in tolerance analysis with ongoing design activities [30]. The 'Toleranced Hybrid' assembly method was developed using CATIAV5, its application programming interface (API) [31] and Python programming language [32]. Its purpose is to provide a means by which the designer can enhance the nominal digital environment through automatic creation of equivalent part models for components at their upper and lower tolerance limits. The access to this information within the digital environment creates a more 'tolerance aware' design as the cumulative effect of tolerances on the overall assembly can be modelled and visualized as the design evolves. The method satisfied five main functional objectives, as follows; 
1) Translate numerical tolerance limits (from the tolerance allocation procedure) to physical parts in CAD these limits have been allocated to satisfy a tolerance limit on the assembly dimension and can be used for future tolerance analysis validation.

2) Use the functionality within CAD software to create the models. This ensures that the method is transferable between platforms having exploited available CAD functions.

3) Ensure that the nominal CAD models remain unchanged- as stated, the DMU is used throughout product development; the master model is used for many downstream processing activities.

4) Allow interaction between nominal and 'worst case' models within the same design environment - the creation of the variational models alongside the nominal models will allow ease of investigation of the assembly in different configurations.

5) Replicate the nominal assembly constraints on the new assembly of variational parts to ensure accurate depiction of the assembly conditions.

\section{Methodology development}

The basic premise of current $\mathrm{CAD}$ practice is that geometry is constructed using $2 \mathrm{D}$ sketches or profiles which are extruded (pad), swept, revolved etc. to give 3D forms. These can be modified using features such as pockets, holes, fillet radii, chamfers etc. to form individual parts with nominal dimensions which are set using CAD parameters. Parts are positioned into an assembly where the relationships between each piece are defined using constraints such as face to face, edge to edge, axis to axis etc. The sequence of actions to create, size and constrain a CAD assembly is recorded in the feature tree. This is displayed in the CAD graphical user interface (GUI) under the product tree, as an assembly of parts or as an assembly of products (sub-assemblies) each with their own part branches. The model/DMU is constructed and saved in the part or assembly files when the model is complete. Parameters and constraints can be changed within the feature tree as a design evolves and the CAD software will update sizes and positional relationships automatically for correctly modelled and constrained geometry as relations/links are maintained between elements.

The tolerance analysis proposed for this work relies on the automatic creation of multiple part versions between maximum and least material conditions for the part - in addition to its nominal form. Although the relationships related to part sizes and positions work well when modifying an assemblage of parts, a problem arises when attempting to create multiple versions of the same part(s), for example, nominal, LMC and MMC forms. Using parameters of the nominal part that control and create the variational geometries will only allow the existence of one condition at a time 
and although these parameters can be identified for control [33] they may not necessarily be suitable to change the shape of the part as would be seen in reality $[34,35]$. CAD systems use a very specific naming convention from the boundary representation (B-Rep) name of geometric elements to the names of bodies and shape features and ultimately the names and numbers of parts and products in an assembly. In order to create representations of variational geometries, this naming convention needed to be understood and a standard convention developed to allow the existence and control of multiple part forms.

\section{a. Scaling function}

The methodology developed in this work used the 'Scaling' function at its core to create the variational (maximum and least material) geometries from the nominal parts. The function does not rely on the parameterization of the part which removes the reliance on understanding how the model has been parameterized, allowing non-developers to use the methodology on any DMU as well as the application of the method to assemblies which did not originate in CATIA. The function allows control over both the size and direction of the effect of the dimensional tolerances under investigation through the definition of the elements to be scaled, the reference from which the scale will be created (a plane, face or point) and the scaling ratio. It satisfies objectives one and two outlined previously. Two main limitations of using the scaling function were identified - (i) the function employed within the 'Part Design' workbench of CATIAV5 created a surface based shape/body and (ii) the function scaled the part as an entire entity. The surface based shape limited the recreation of assembly constraints as these are only possible between geometric elements of a volume shape/body. The introduction of the hybrid body in the Generative Shape Design (GSD) workbench overcame this limitation.

\section{b. Hybrid body}

The 'hybrid body' as named by CATIA, in the GSD workbench, permits the creation of geometric elements within a 'Geometrical Set' branch of the product tree. The GSD workbench supports creation of wireframe construction elements during preliminary design and enrich existing 3D mechanical part design. It is a complement to Part Design and supports requirements of solid-based hybrid modeling ${ }^{\dagger}$. The hybrid body, created as a replica of the nominal part with its shapes (individual or multiple) scaled accordingly resulted in the creation of a hybrid body volume. The elements of this volume body are available for constraint creation. This facilitated the creation of the variational

\footnotetext{
${ }^{\dagger} \mathrm{http}: / /$ catiadoc.free.fr/online/CATIAfr_C2/sdgugCATIAfrs.htm
} 
geometries alongside the nominal part, as the hybrid body and part body can co-exist in the same environment, as well as allowing the creation of forms between the MMC and LMC within the same hybrid body, so only a single addition to the part file is required to create two (or more) representations of the variational geometry. As well as this, the features/shapes of the hybrid body can be scaled individually or as a group to create the desired variational geometry. This satisfied objectives two to four outlined previously and ensured that objective five is supported.

The methodology was developed based on a simple 'Wheel Mount' [28] which is an assembly of 3D rectangular blocks, see Fig. 1. These were created in CAD using extruded 2D sketches. The blocks each have a single round hole and they are assembled/held together using a cylindrical pin and bolt. Having established the basic framework for exploiting multiple versions of the 3D geometry to conduct tolerance analysis, the methodology was developed and refined using elements of the Engine assembly shown in Fig. 4. In this case the geometry was more complex and the functions used to develop the various shapes and features included pad, revolve and pocket presenting opportunities to enhance the method to support these complex industrial products and determine the applicability of this method on a more advanced CAD assembly.

The methodology consists of two main aspects; 1 . Creation of the variational geometry and 2 . The regeneration of the nominal assembly conditions, Fig.3. Friel et al. [28] describe the method as developed on the simple block assembly; each part is modeled as variational geometry according to their respective maximum and minimum tolerance dimensions using the method outlined previously. 
USER

\section{PYTHON}

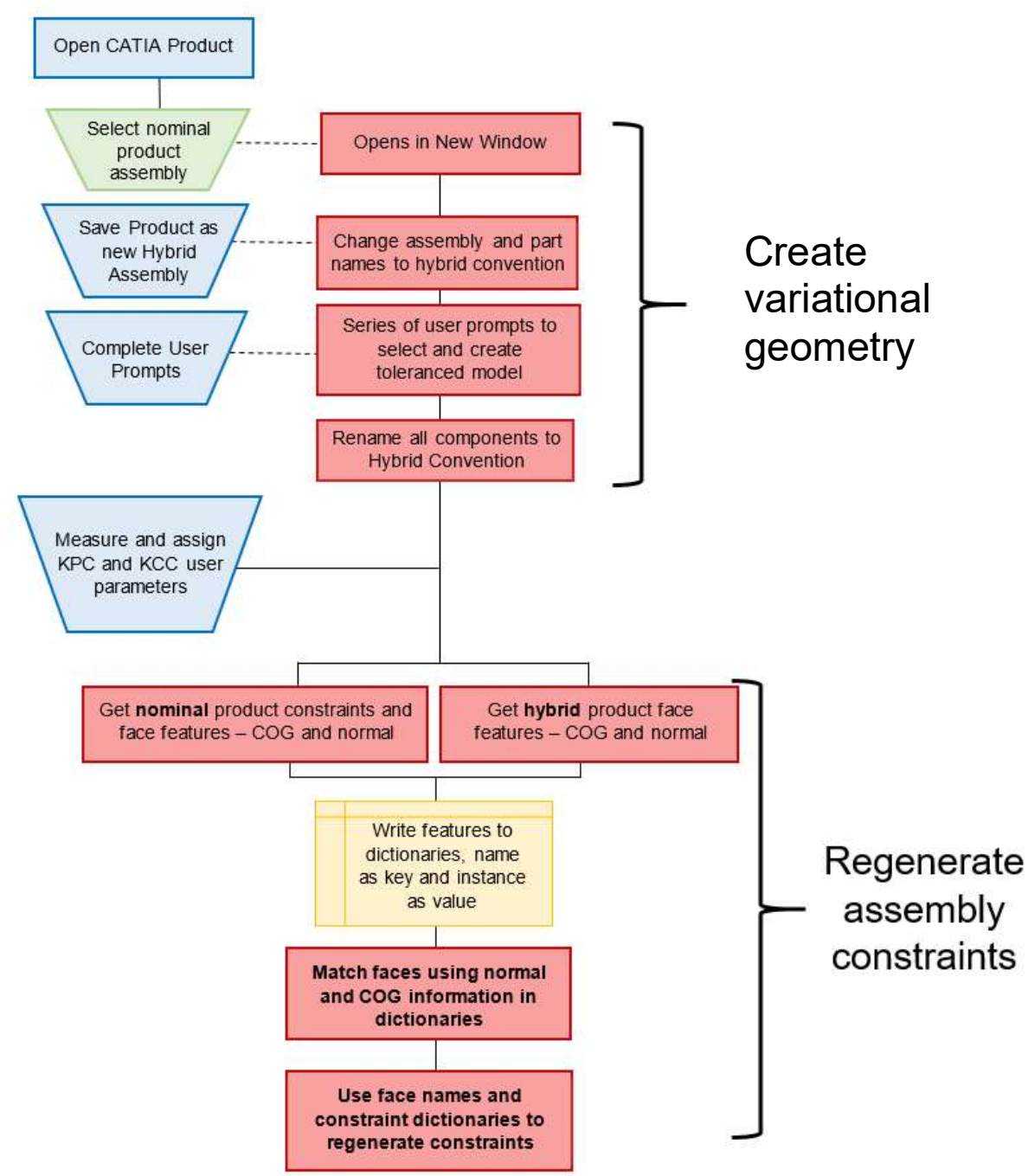

\section{Fig. 3 Toleranced Hybrid Assembly Creation Process}

In order to respect the nominal assembly conditions, the routine to regenerate the assembly constraints is employed. This routine was developed to equivalence the faces of the nominal 'Part Body' involved in the surface contact constraint with those in the 'Hybrid Body' by matching the normal vector and center of gravity (COG) of the face element. This face pair was then used to match the nominal face element with that used in the surface contact constraint to create the new constraint on the assembly using the equivalent hybrid face. The surface contact constraints are accurately regenerated on the Toleranced Hybrid simple block assembly, which propagates the dimensional effect of the variational geometries on the final assembly conditions. All methodology aims were satisfied. 


\section{Methodology refinement}

The methodology developed in Section III.B.1 was employed and subsequently refined on the Piston Crankshaft sub-assembly within an Internal Combustion Engine assembly, Fig. 4. This model provided a more geometrically complex example on which to employ the method and exploit it for tolerance analysis purposes. The parts themselves were more complex in shape than those of the simple block assembly and thus expanded the functionality beyond its simpler block type geometry. As well as this, surface contact constraints and coincident constraints exist on the nominal Engine assembly, once again, expanding the methodology to support the regeneration of both of these constraint types.

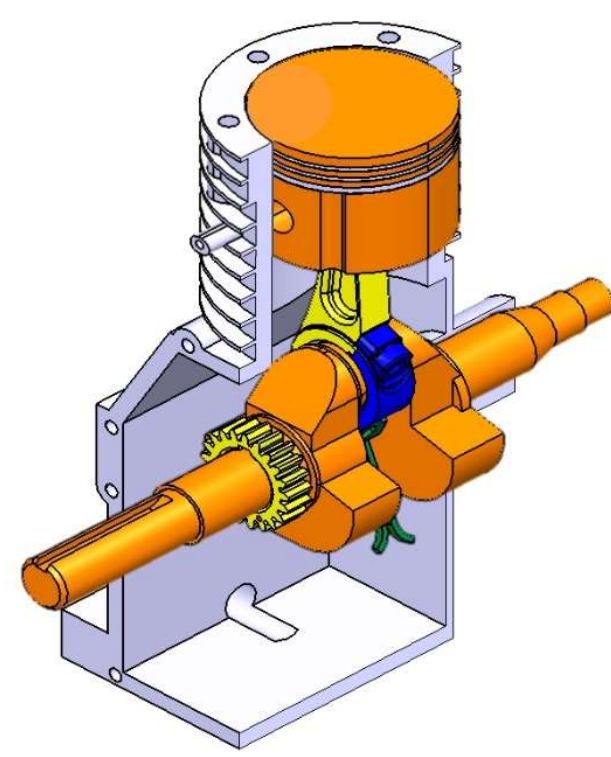

Fig. 4 Piston Crankshaft Sub-Assembly

\section{a. Hybrid body creation}

The creation of the complex shapes in the Engine assembly involved more design operations that the simple 'Pad' volumes of those in the simple block assembly. As is commonplace in design, to create complex shapes, multiple operators are used to completely define the shapes of the part. In the Crankshaft for example, the spindle and axle, Fig. 5 are revolved sketches with pockets creating the slotted features, whereas other shapes, such as the shoulder, are created using extruded volumes with pad volumes removed to create the final shape. As the work presented here creates a replica of the nominal form in the hybrid body, the functionality was expanded to support the translation of these operators in Part Design (pad, revolve, remove etc.) to their equivalent operations in the GSD workbench with 
its own specific surface, volume and finishing operators. This supports the automation of the routine in the CAD environment. As previously introduced the scaling function in the GSD workbench, performed on the new replica of the nominal part, permits the creation of both scaled shapes (single features) of the part, e.g. the spindle of the Crankshaft, Fig.5, as the hybrid body recognizes individual feature shapes rather than the body as a whole, a limitation of using the scaling function within the Part Design workbench or the scaling of the entire part entity, e.g. the Piston Connecting Rod, Fig.6. The creation of either or both of these in the hybrid body required, once again, a standard naming convention, and a method to control the presence of the relevant part shapes. For example, during the creation of a scaled feature in the hybrid body, the default name 'Scaling.1' does not make the user aware of the representation of which form the part is in. Thus, the automatic creation of a standard naming convention based on the user's input, defines the new scale shape as either 'PartName\%Scaling.1_MMC' or 'PartName\%Scaling.1_LMC'. This naming convention, and the separating values, (\% or _ ) are required during the constraint regeneration to identify and differentiate the part in question and the variational geometry in question.

Another control feature of the method was developed as a result of the need to scale the entire part, Fig.6. As the input to the function was the selection of multiple features, so too was the output - a 'Multi-Output' of scaled shapes. This multi-output feature required its own handling procedure - including its specific naming convention and identification of the input 'parent shapes' so as to establish those feature shapes which are involved in the representation of the entire variational geometry for future representation and constraint regeneration. Finally, the hybrid body is a new geometrical entity within the part with its own branch in the tree structure and is dissociated from its part body, therefore, the constraints associated to the elements of the Part Body are not maintained when the new hybrid body is created. This requires, once again the equivalencing of geometric elements to support the regeneration of assembly constraints in order to accurately propagate the effect of the variational geometries on the final assembly conditions. 


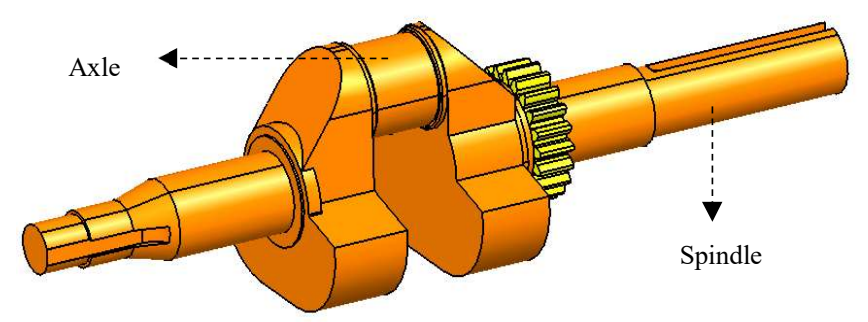

Fig. 5 Crankshaft Axle and Spindle

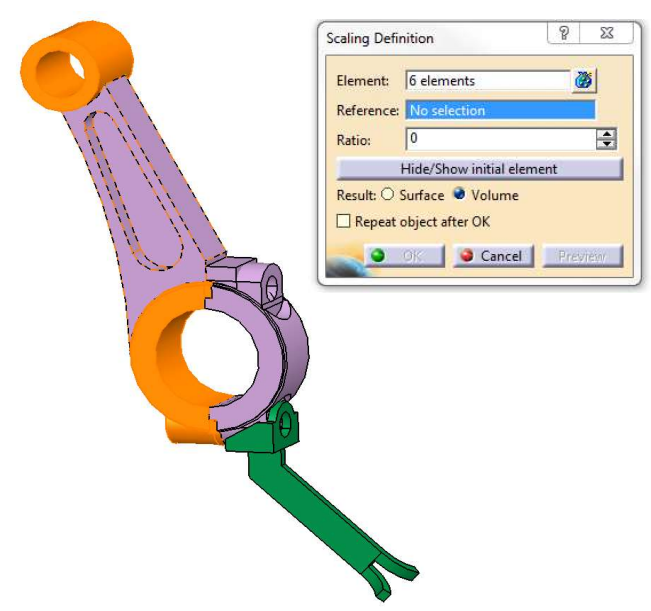

Fig. 6 Piston Connecting Rod - Shape Selection for scaling the entire hybrid body

\section{b. Constraint regeneration}

The latter part of the method which is to recreate the nominal assembly constraints on the new assembly of variational geometries has been expanded on this assembly product to include regeneration of both surface and coincident constraints. Surface contact constraints between face elements have previously been equivalenced by matching surface normal vector and COG co-ordinates iteratively between the faces of all the parts. In a simple assembly with a small number of parts, this is computationally viable however, as the number and complexity of parts increases so too does the number of elements to compare. Thus a more efficient method, the k-d tree 36] was introduced in the Engine assembly to equivalence the thousands of geometric elements. K-d tree or k-dimensional tree is a data structure for organizing some number of points in a space with k-dimensions. In this case, the k-d tree contains the center of gravity dimensions (COG; $(\mathrm{x} 1, \mathrm{y} 1, \mathrm{z} 1))$ of every planar face and another contains the axis midpoint $(\mathrm{x} 1$, $y 1, z 1)$ of every axis in every part within the assembly, so both are in 3D space. From the k-d tree which stores these values, a 'nearest neighbor' search function [37] using the COG or axis points of the hybrid face in question as the input, will return the location (as an integer) within the input array used to create the $\mathrm{k}-\mathrm{d}$ tree of the nearest neighboring face. This location result must be translated to a face B-Rep name so as to equivalence the faces by name and create a new constraint using this name. Thus a referencing dictionary developed concurrently with the k-d tree input array, will return the B-Rep dictionary 'value' of the nominal face, given the key (integer) matches the location integer returned during the nearest neighbor search. With this element pair, whether it be a face match or axis match the respective contact or coincident constraint can be regenerated on the Toleranced Hybrid assembly. Using the k-d tree 
as an alternative equivalencing method to that previously employed on the simple block assembly resulted in a $61 \%$ reduction in run time. Once the element pairs are created the constraint creation takes a matter of seconds.

Figure 7 shows the final methodology for the creation of the Toleranced Hybrid assembly based on the previous description. It depicts the two main processes for the creation of the variational parts and the regeneration of the assembly constraints. It also shows how the data extracted for element equivalencing is managed within Python using function specific dictionaries. This data structure is a means in which to store the instances of constraints, geometric elements B-Rep names and instances alongside their COG or axis mid-point value.

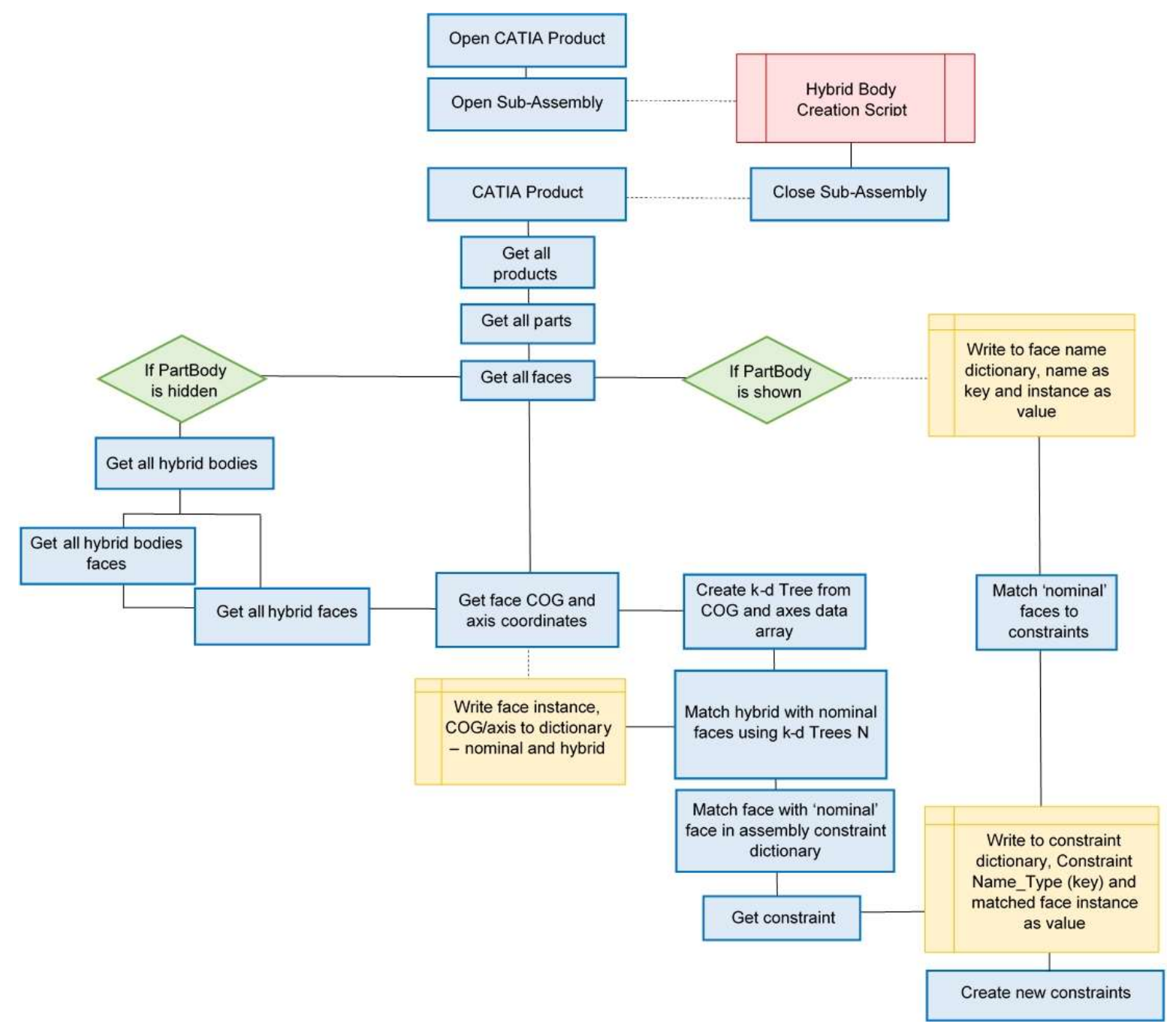

Fig. 7 Flowchart of Toleranced Hybrid Assembly script 


\section{IV.Exploiting the enriched DMU}

This section describes the exploitation of the enriched DMU for analysis purposes. The simple block assembly example is used for worst-case tolerance analysis, the preliminary and simplest form of tolerance analysis performed during product development. It calculates the cumulative effect of the contributing dimension tolerances on a key product dimension to determine its tolerance limits. At the early stages of design this will begin to describe the tolerancing scheme on the assembly and allow some manufacturing perspective within the design domain. As design is almost exclusively performed within the CAD environment, paper based worst-case analysis is rarely if ever performed. The 'Toleranced Hybrid' assembly methodology developed here can support tolerance analysis during design.

The Engine assembly demonstrates how the methodology and analysis can be applied to an industrial example containing more complex and varied parts. An analysis of the effects of a single part in MMC and LMC conditions on a critical to function dimension demonstrates the successful implementation of the enriched DMU with configuration analysis. In engineering terms, it shows how the enriched DMU can facilitate understanding of the tolerance stack-up in the CAD domain.

The Aircraft Stiffened Panel is another example of an industrial product, this time within the aerospace domain which is strictly governed by precise tolerances. The enriched DMU of this product has been exploited to understand how variation of part dimensions within a predefined assembly sequence has an effect on the quality of the assembly and the ultimate function of a product.

It is pertinent to note at this point that the methodology and analysis begins with $\mathrm{KCC}$ values (modelled as variational geometry) and calculates the corresponding KPC value based on whichever combination of variational geometry is being analysed. The configuration analyses herein validate the numerical values of the tolerance analyses from literature and demonstrate that the methodology is capable of performing worst case tolerance analysis.

\section{A. Simple Block Assembly}

Using the Toleranced Hybrid simple block assembly a script has been developed to perform worst-case and configuration analysis. The purpose of this analysis is to perform classical worst-case tolerance analysis on all potential configurations of the assembly given the material conditions of the parts to determine a complete tolerance analysis picture. It is the aim of this work to create a means by which the design engineer can perform quick analysis for initial 
tolerance specification on the assembly, gain an understanding of the effect of tolerances on the assembly function and identify the important contributors to assembly tolerance for ongoing design work.

\section{DMU setup}

The assembly is configured within CATIA to represent the tolerance dimensions calculated by Wan et al. [7] from a tolerance allocation procedure. However, these tolerances could be from different sources, manufacturing process tolerances, standards, even estimated tolerances for investigative purposes. The published tolerances have been chosen to validate the results of the configuration analysis. Friel et al. [14] describe the employment of the method to create the Toleranced Hybrid assembly.

Finally, to interpret the results of the configuration analysis it is important to record the dimensional changes in the key characteristic dimensions, all KCC and KPC dimensions. This requires creating measurements of these dimensions between all the faces involved in the measurement, e.g. KPC1, is measured between two nominal faces, but it requires new measurements between the faces in the hybrid body as the faces are dissociated from the nominal part and therefore their respective measurement. Once completed these measures are translated to parameters to be extracted during the analysis.

\section{Configuration analysis procedure}

The routine developed is automated to support the design engineer without additional workload. It consists of three main activities, 1. Data extraction, 2. Configuration generation and 3. Result recording. The script reads the Toleranced Hybrid assembly for complete extraction of information relevant to the analysis. This includes the conditions of all parts of the assembly, whether they are nominal or variational using the developed methodology, i.e. presence of any hybrid body with MMC/LMC or both shapes. Using this information a Cartesian product of the part conditions creates all possible configurations of the assembly, in this simple block assembly case there are 243 different configurations. The configuration specific assembly constraints must be regenerated on the assembly, using the procedure outlined previously. Finally the script reads the $\mathrm{KC}$ dimensions and writes to an output file alongside the assembly configuration information. The configured assembly is reset to the original nominal configuration before the next analysis is performed. This process is repeated for each configuration, taking 14 minutes using a $3.4 \mathrm{GHz}$ CPU. 


\section{Results}

Initial results facilitate the preliminary tolerance specification for the KPCs of the assembly. Two hundred and forty three configuration results were generated for this assembly and stored in an output file. The largest dimensional change recorded on both the KPCs happens at two configurations, Fig.8. This is representative of the upper and lower tolerance limits of the KPC dimensions as calculated in the tolerance analysis by Wan Din et al [7].

\begin{tabular}{|c|c|c|c|c|c|c|}
\hline Part1.1 & Part2.1 & F Part3.1 & - Part4.1 & Part6.1 & П KPC1 & C2 $\mathrm{T}$ \\
\hline Part1_MMC & Part2_MMC & Part3_MMC & Part4_LMC & Part6_LMC & 0.7 & -0.7 \\
\hline Part1_LMC & Part2_LMC & Part3_LMC & Part4_MMC & Part6_MMC & -0.7 & 0.7 \\
\hline
\end{tabular}

Fig. 8 Assembly configurations showing KPCs at their extreme tolerance limits

It has been noted that these extreme tolerance limits on the KPC dimensions are not experienced when the assembly is in the 'worst-case' conditions, i.e. all parts at LMC or MMC conditions. Rather, a combination of material conditions resulted in these maximum limits, Fig.8. At 'worst-case' conditions the tolerance limits on the KPC dimensions are shown in Fig.9. This shows the importance of performing a complete tolerance analysis procedure for all assembly configurations to determine a more accurate specification of the KPC tolerance limits going forward with design.

\begin{tabular}{|c|c|c|c|c|c|}
\hline Part1.1 & F Part2.1 & Tr Part3.1 & F Part4.1 & TI Part6.1 & F $K P C 1$ - KPC2 \\
\hline Part1_MMC & Part2_MMC & Part3_MMC & Part4_MMC & Part6_MMC & -0.5 \\
\hline Part1.1 & NT Part2.1 & N. Part3.1 & N Part4.1 & F Part6.1 & PC1 $F$ KPC2 \\
\hline Part1_LMC & Part2_LMC & Part3_LMC & Part4_LMC & Part6_LMC & -0.1 \\
\hline
\end{tabular}

Fig. 9 'Worst case' configuration results

\section{Validation}

This analysis has been derived from a tolerance allocation example [7] where the KPC tolerances were known. These tolerance limits were confirmed on the assembly from the tolerance analysis results, which indicate that these are in fact the maximum deviation experienced by the KPC dimensions, Fig.8, given the tolerances modeled on the parts. The analysis also provides the configuration which defines these tolerance limits. The Toleranced Hybrid assembly method supports the creation of variational geometries from any give tolerance value, which is in the control of the designer, meaning that multiple analysis of different tolerance values can be performed and compared, a useful insight for product development engineers, without the use of third party software. 


\section{B. Engine Assembly}

Figure 10 shows the key characteristics on the Piston Crankshaft sub-assembly of an internal combustion engine, with the subsequent tolerance functional equation shown by Eq. (2), adapted from Mao et al.[38]. This has been validated by the sensitivity analysis procedure [7]. The Piston and Crankshaft is a critical to function sub-assembly of the engine as the movement of the Crankshaft and Piston controls the size of the gap between the piston top surface and the head assembly. This gap, KPC1 in Fig.10, must be controlled in order for the engine to function efficiently. Any dimensional changes in the parts of the assembly will have an effect on the KCCs and the KPCs, ultimately affecting the engine efficiency - product functionality and quality.

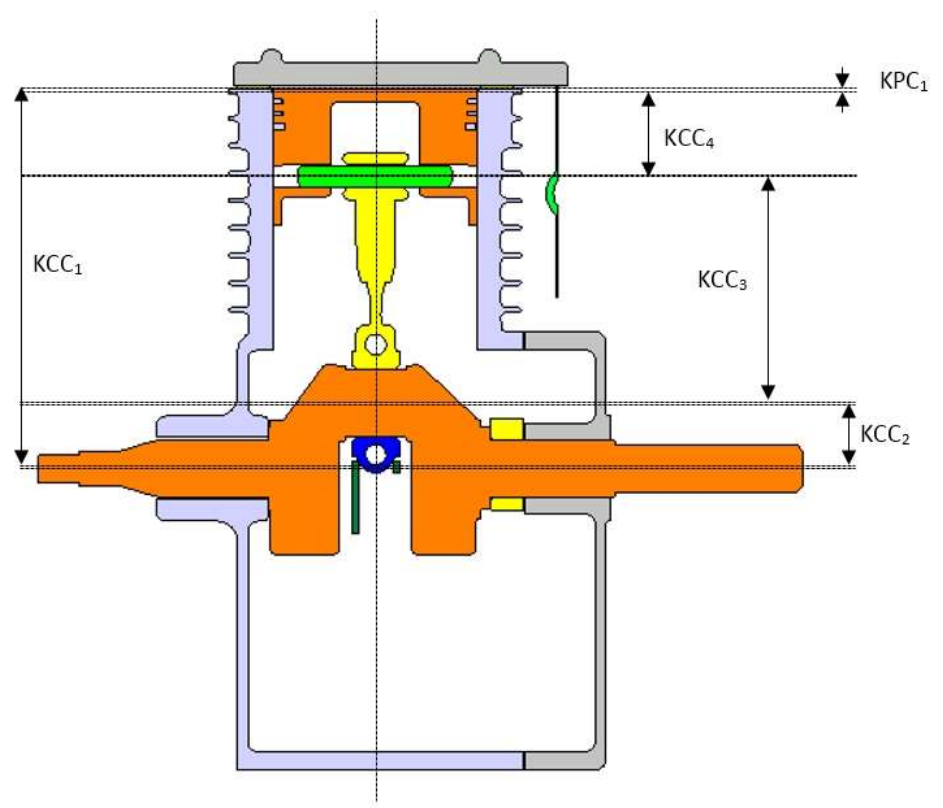

Fig.10 Assembly draft of Engine showing $\mathrm{KC}$ definition

\section{DMU setup}

The sub-assembly parts have been sized according to manufacturing process specific tolerances 38 ] using the Toleranced Hybrid Assembly process to determine the impact of dimensional changes of the piston connecting rod and crankshaft on the overall dimension of critical gap, KPC1. The Piston Connecting Rod, Fig.6, is a cast aluminum part, which has been scaled from the center point, to represent the casting process tolerance on the vertical dimension, KCC3. The tolerance value has been taken from ISO 2768-.1 1989 [39] - the toleranced dimension is $72.136 \pm 3.2$ $\mathrm{mm}$. The methodology supports the creation of this scaled part in this way through the selection of all shapes as reference for the scaling function discussed in Section III.B.2.a. Conversely the methodology also supports the 
separate tolerancing of features of the part, for example, the crankshaft axle and spindle features can be scaled as separate entities to represent different tolerance grades.

\section{Configuration analysis}

The Engine assembly was analyzed in the same manner as the simple block assembly, with the data extraction process reading and storing the data for all nominal and hybrid part bodies, recognizing the presence of MMC and LMC parts. Each possible configuration of the Engine with the non-nominal parts was represented in the CAD environment, with configuration specific constraints replicating the nominal assembly conditions on the new assembly. The KPC measurement, i.e. the critical to function dimension was recorded at each configuration alongside the specific configuration of parts.

\section{Results}

The results are shown in Fig. 11. For visualization purposes the configuration of the other parts has been excluded as they all exist in the nominal form. This simple analysis demonstrated the successful implementation of the methodology and analysis on a complex industrial product. The results show how the inclusion of variational geometries within a nominal assembly can provide more realistic representation of the assembly containing 'as manufactured' parts. Nine of the twenty seven configurations of the assembly (specific to this analysis) are shown in Fig.11. The resulting KPC dimension given the specific configuration is also shown, defining the dimensional changes as a direct result of the MMC and LMC Piston Connecting Rod. The dimension has increased and decreased in size from the nominal dimension of $1.04589 \mathrm{~mm}$ given the presence of the Piston Connecting Rod in its variational form. The analysis can also support the analysis of multiple parts under different tolerance dimensions and the inclusion of more parts in their variational form would contribute to the tolerance stack-up and ultimately the KPC dimension. The run time for this simple analysis was 197 minutes using a 3.4GHz CPU. 


\begin{tabular}{|c|c|c|c|c|c|c|c|}
\hline Crankshaft.4 & Piston_Connecting_Rod.16 & Piston_Rod_Cap.7 & KCC1 & KCC3 & KCC5 & KCC7 & KPC1 \\
\hline MMC & MMC & MMC & 0 & 0 & -1.72122 & 0 & 0.31609 \\
MMC & MMC & LMC & 0 & 0 & -1.72122 & 0 & 0.31609 \\
MMC & MMC & Nominal & 0 & 0 & -1.72122 & 0 & 0.31609 \\
MMC & LMC & MMC & 0 & 0 & -3.30483 & 0 & -3.50855 \\
MMC & LMC & LMC & 0 & 0 & -3.30483 & 0 & -3.50855 \\
MMC & LMC & Nominal & 0 & 0 & -3.30483 & 0 & -3.50855 \\
MMC & Nominal & MMC & 0 & 0 & 0.0613 & 0 & 0 \\
MMC & Nominal & LMC & 0 & 0 & -0.82374 & 0 & 0 \\
MMC & Nominal & Nominal & 0 & 0 & 0 & 0 & 0 \\
\hline
\end{tabular}

Fig. 11 Results of configuration analysis on Engine assembly showing dimensional change in KPC as a result

\section{of variational part forms}

\section{Stiffened Aircraft Panel}

Figure 12 shows a Stiffened Aircraft Panel, a structural part within an aircraft fuselage. There are eighteen parts in this assembly, seven vertical diaphragms including two uplocks, the skin panel, six horizontal structural support beams, three gussets and a bracket. Uplock and diaphragm parts are assembled on to a skin panel, with structural beams connecting these, providing structural integrity. It is important during assembly that all of these parts are correctly fitted to ensure assembly function as well as maintaining its structural integrity. When developed in CAD, the nominal models produce an assembly where all parts fit perfectly with no part gaps.

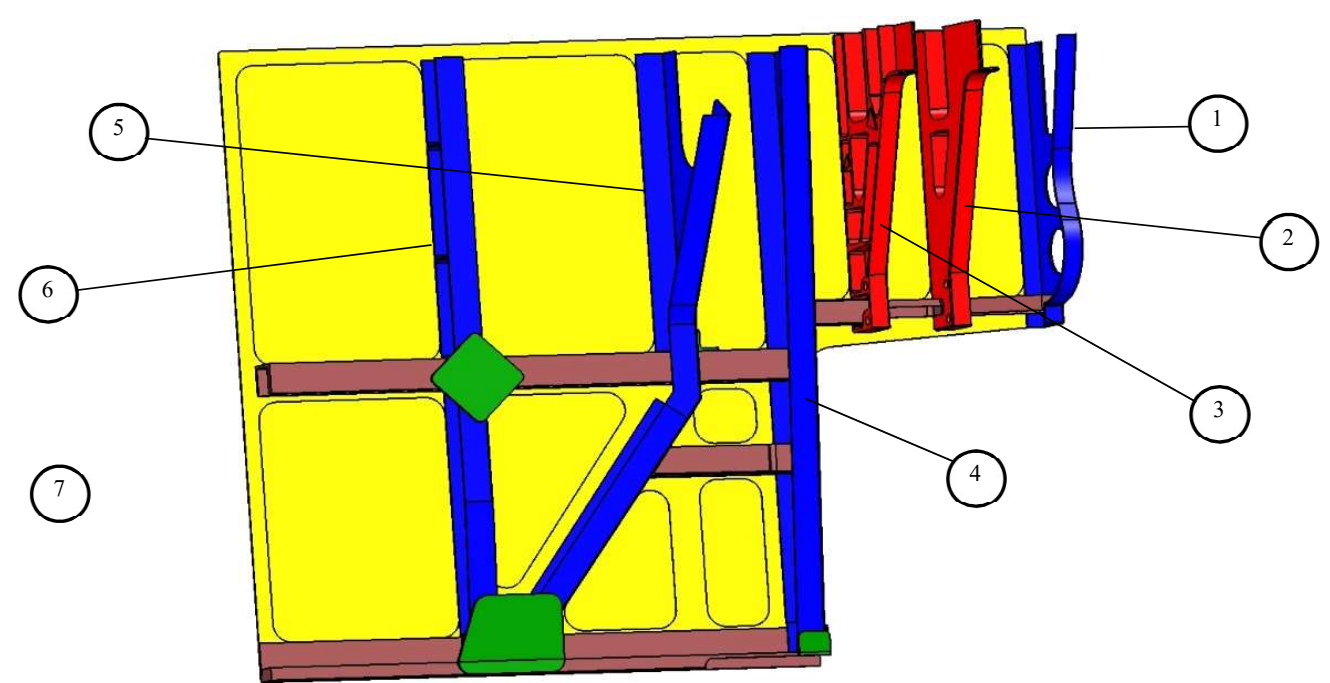

Fig. 12 Stiffened Aircraft Panel 


\section{DMU setup}

The established assembly sequence for this product was investigated to determine how the inclusion of the variational parts within the assembly affect its key dimensions. To ensure that for each configuration the constraints are regenerated in the order of the assembly sequence they have been numbered sequentially. This is controlled within the script routine.

The dimension of interest in this particular analysis was the gap between Diaphragm B and Beam D, Fig. 13. As a structural beam, this gap must be kept to a minimum (i.e. within a tolerance) to ensure proper contact between the surfaces. The procedure supports the analysis of multiple dimensions, as shown in the simple block assembly example, however, for this example, only one dimension was investigated.

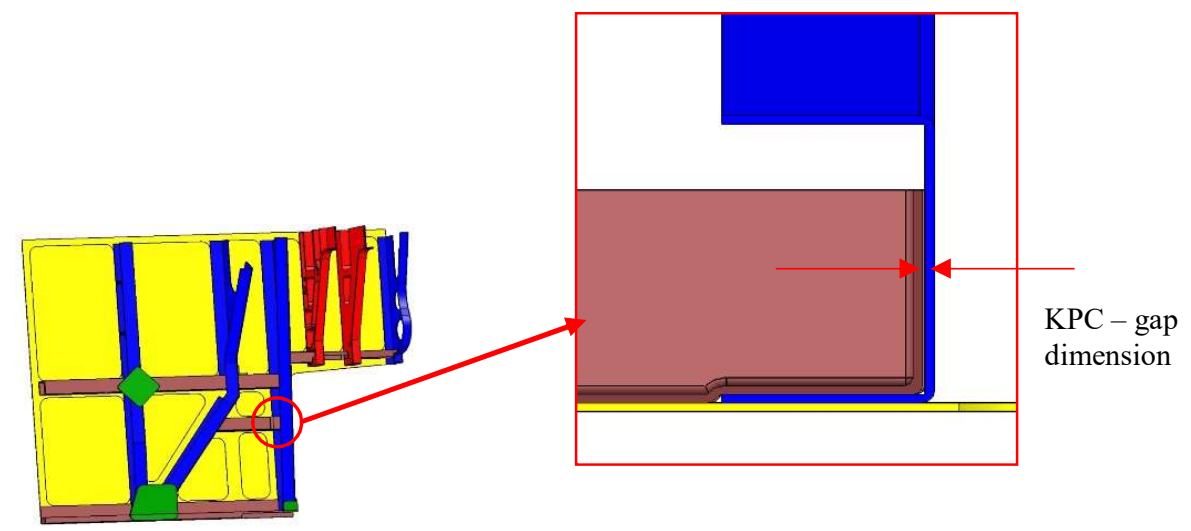

Fig. 13 KPC gap dimension under investigation

Once again the parts have been modeled according to tolerance standards (ISO 2768) for specific manufacturing processes. Table 2 shows the parts, their nominal dimension (in the direction of tolerance stack-up), and their manufacturing process tolerance (according to the standard). This information was used to create the Toleranced Hybrid assembly from which the configuration analysis was performed.

Table 2 Stiffened panel parts and dimensions to be toleranced

\begin{tabular}{ccccc}
\hline \hline $\begin{array}{c}\text { Bubble } \\
\text { Number } \\
\text { (Fig.12) }\end{array}$ & Part & Manufacturing process & $\begin{array}{c}\text { Nominal } \\
\text { dimension } \\
(\mathbf{m m})\end{array}$ & Tolerance (mm) \\
\hline 1 & Diaphragm A & CNC machined - milled & 30 & 0.1428 \\
2 & Uplock Fwd & CNC machined - milled & 30 & 0.1428 \\
3 & Uplock Aft & CNC machined - milled & 30 & 0.1428 \\
4 & Diaphragm B & CNC machined - milled & 45 & 0.1646 \\
5 & Dog Leg & Formed - folded & 45 & 0.1646 \\
6 & Top Rib & CNC machined - milled & 45 & 0.1646 \\
7 & Bottom Rib & CNC machined - milled & 45 & 0.1646 \\
\hline \hline
\end{tabular}




\section{Configuration analysis}

In this product seven parts were modeled in the variational form, resulting in fourteen hybrid bodies alongside their nominal counterparts. This number of variational parts in an assembly of eighteen parts resulted in 2187 possible configurations for analysis. The analysis ran each configuration at an average of 1.5 minutes per configuration. The total run time for this part was in the range of 54 hours.

\section{Results}

Figure 14 shows a small range of the result data, showing the configurations which resulted in the largest dimensional changes in the KPC dimension under investigation. This dimension was a gap between two faces, Fig. 13 . A positive dimensional change indicates a gap appearing between the parts, whilst a negative dimension changes indicates interference between the parts. In reality interference between the parts means resistance to fit and could result in part rework or scrap. For large gaps appearing between parts, the gap will require shimming, an extra manufacturing process for the supplier at a cost to them for both the shimming process and potential weight penalty by the customer.

These results give the user an understanding of the effect of the variational parts on critical dimensions, for example, both uplocks in MMC condition, along with Diaphragm B and the Dog Leg in their MMC condition results in the largest dimensional change in the KPC, i.e. large interference or resistance to fit between the structural Beam D and Diaphragm B. With knowledge like this the designer can focus efforts on particular parts, for redesign or process change or indeed, investigate assembly sequence alternatives to determine an optimum with respect to this key product characteristic or others.

\begin{tabular}{|l|l|l|l|l|l|l|r|}
\hline UplockAft & UplockFwd & DiaphragmA & DiaphragmB & DogLeg & TopRib & BottomRib & KPC1 \\
\hline MMC & MMC & MMC & MMC & MMC & Nominal & Nominal & -3.07416 \\
MMC & LMC & LMC & MMC & MMC & LMC & Nominal & -2.00962 \\
MMC & Nominal & MMC & MMC & MMC & Nominal & Nominal & -3.001 \\
LMC & LMC & LMC & Nominal & LMC & MMC & LMC & 0.7499 \\
Nominal & MMC & Nominal & LMC & LMC & Nominal & Nominal & 10324 \\
Nominal & MMC & Nominal & LMC & Nominal & MMC & MMC & 1.00381 \\
\hline
\end{tabular}

Fig.14 A selection of results from the stiffened panel analysis 


\section{V.Discussion and Conclusions}

This paper has identified from literature the potential to exploit CAD software for more tolerance aware product development. CAD systems operate based on the concept of nominal modeling which produces parts of perfect dimensions and products of exact assembly dimensions. In real life however, manufacturing results in, more often than not, dimensional variation of parts. This variation has been excluded from the product design process, being analyzed effectively during later stages of product development using a highly defined CAD model, with established assembly processes and tolerances in third party computer software (CAT). Furthermore, current practice sees tolerance information appended to models as annotation as this is the only functionality available in commercial CAD systems. This work identified the absence of functionality to support the creation of multiple versions of the same part as a means to model variational geometry. In fact, creating new geometries within the same part using available functions in the design workbench meant that relationships and identifications between the same parts within the DMU were broken. This greatly informed the methodology to ensure robust modelling of variational geometries that were integral to the DMU.

The presence of the variational geometries in the hybrid assembly means tangible models representing 'as manufactured' parts are stored within the DMU for analysis as early design and as design progresses. This focuses design and designers on the manufacturing issues that arise during production that are 'invisible' at the design stage and thus remain unaccounted for. This method brings tolerance awareness more firmly into the design domain allowing for preliminary tolerance analysis within the CAD environment.

The methodology established and presented in this paper supports the creation and analysis of variational part forms alongside their nominal counterparts, enriching the DMU for investigations into tolerance and assembly analysis based on the inclusion of these non-variational forms. The methodology was developed with five main objectives in mind, to represent tolerance limits as physical parts in $\mathrm{CAD}$, using the CAD functionality and ensuring nominal models remain unchanged, support the co-existence of part forms in the same environment and ensure nominal assembly conditions are regenerated on any variational assembly conditions. Further refinements of the method included the employment of the k-d tree to more accurately and faster search for and equivalence faces, sparing computational expense.

The enriched DMU has been exploited using three assembly examples and with varying model complexity and structure. The results show how the enriched DMU can be used for preliminary tolerance analysis to define tolerances 
on the KPC dimension of the assembly, based on the representation of the parts in their respective variational forms. The simple block assembly example demonstrates this capability based on the published tolerance allocation of the KPC limit on the contributing KCCs. The approach is still applicable in the absence defined part tolerances and can be used as an investigation in to tolerance analysis during early stage design.

The Engine assembly provided an example to refine the method and successfully employ the dimensional analysis on a more complex industrial product. The Piston Crankshaft example results show how assigning and modeling dimensional variation based on toleranced standards can inform the designer of the effect this tolerance has on the KPC dimension. Further investigation of other parts in their variational form based on various manufacturing processes could provide a comparative investigation in to the effect of process tolerances on product functionality.

The Aircraft Panel investigation showed that the perfect assembly conditions of the nominal parts are indeed affected by the inclusion and analysis of variational part forms, providing numerical results indicating the presence and extent of the dimensional effect. The predefined assembly sequence was recreated on each variational assembly configuration with the resultant measure indicating clash or interference between structurally integral parts. This informs the designer of the need to adjust the assembly sequence or review the manufacturing process if this was a conceptual product. It must be noted that for all of these examples, and the development of the methodology, only dimensional variation is supported. Geometric deviation also impacts the quality and assembly of parts and the effect of tolerance stack-up and will be the subject of future work.

The methodology and examples have been developed and analyzed using CATIAV5 and Python programming language. It is applicable in other CAD software using other languages based on the existence of equivalent functions to create clones or hybrids of parts combined with the API functionality and methods used to regenerate assembly constraints. The methodology has been developed to be independent of a parameterized model, through the use of the scaling function. This means the method is compatible with so called 'dumb solids' where the feature tree is not available e.g. as a result of a file transfer via Step, although not explored in this work, is a topic for future research.

In conclusion, the methodology presented here has extended the capability of commercial CAD software beyond its current use to better inform design from a manufacturing perspective by addressing and overcoming the limitations of $\mathrm{CAD}$ as a nominal product modeler. Irrespective of CAD maturity, there is a requirement to better equip designers to make decisions based on the best available manufacturing information. This methodology provides the technology to do this and a key benefit is the consideration of tolerance stack-ups by designers as soon as individual parts are 
modelled and digitally assembled into 'part to part' fits and sub-assemblies. The information is subsequently stored in the DMU making tolerance stack up intrinsic to the whole product development process - starting at, but not limited to, the early design stage. With this new functionality, protocols need to be established in the industrial domain to exploit this method with respect to more complex assemblies (such as whole aircraft) while respecting current practices around process capability definition. Through engagement with industry the added value of this work could be quantified through lead time estimates and online production quality checks before and after implementation of this methodology. 


\section{$\underline{\text { References }}$}

[1] Kang, X., and Peng, Q., "Integration of CAD models with product assembly planning in a Web-based 3D visualized environment," International Journal on Interactive Design and Manufacturing, Vol. 8, No. 2, 2014, pp. $121-131$

doi: $10.1007 / \mathrm{s} 12008-014-0220-9$

[2] Wang, C., Bi, Z., and Xu, L.D., "IoT and cloud computing in automation of assembly modeling systems," IEEE Transactions on Industrial Informatics, Vol. 10, No. 2, 2014, pp. 1426-1434. doi: 10.1109/TII.2014.2300346

[3] Senaltun, G., and Cangelir, C., "DMU management-product structure and master geometry correlation," 20th ISPE International Conference on Concurrent Engineering, CE 2013 - Proceedings, 2013, pp. 353-360.

[4] Danesi, F., Gardan, N., and Gardan, Y., "Collaborative design: From concept to application," Geometric Modeling and Imaging New Trends, Vol. 2006, 2006, pp. 90-96.

[5] Sivakumar, S., and Dhanalakshmi, V., "An approach towards the integration of CAD/CAM/CAI through STEP file using feature extraction for cylindrical parts," International Journal of Computer Integrated Manufacturing, Vol. 26, No. 6, 2013, pp. 561-570. doi: 10.1080/0951192X.2012.749527

[6] Boussuge, F., Shahwan, A., Léon, J., "Template-based Geometric Transformations of a Functionally Enriched DMU into FE Assembly Models," Computer-Aided Design and Applications, Vol. 11, No. 4, 2014, pp. 436-449. doi: $10.1080 / 16864360.2014 .881187$

[7] Wan Din, W.I., Robinson, T.T., Armstrong, C.G., "Using CAD parameter sensitivities for stack-up tolerance allocation," International Journal on Interactive Design and Manufacturing (IJIDeM), Vol. 10, No. 2, 2014, pp. $139-151$

doi: $10.1007 / \mathrm{s} 12008-014-0235-2$

[8] Dolezal, W.R., "Success factors for Digital Mock-Ups (DMU) in complex aerospace product development," Thesis, 2008,

[9] Zhang, H., and Li, J., "Modeling method and application in digital mockup system towards mechanical product," Advanced Materials Research, Vol. 605-607, 2013, pp. 604-608. doi: 10.4028/www.scientific.net/AMR.605-607.604 
[10] Glende, W.L., "The Boeing 777: A Look Back," AGARD FVP Symposium, 1997.

[11] Maropoulos, P.G., and Ceglarek, D., "Design verification and validation in product lifecycle," CIRP Annals Manufacturing Technology, Vol. 59, No. 2, 2010, pp. 740-759. doi: $10.1016 /$ j.cirp.2010.05.005

[12] Foucault, G., Shahwan, A., Léon, J., "What is the content of a DMU? Analysis and proposal of improvements," AIP-PRIMECA 2011 - Produits, Procédés et Systèmes Industriels : intégration Réel-Virtuel, Le Mont Dore, France, 2011.

doi: 10.1016/j.cad.2012.10.021

[13] Shahwan, A., Léon, J., Foucault, G., "Qualitative behavioral reasoning from components' interfaces to components' functions for DMU adaption to FE analyses," Computer-Aided Design, Vol. 45, No. 2, 2013, pp. 383-394.

doi: 10.1016/j.cad.2012.10.021

[14] Nolan, D., "Defining Simulation Intent," Queen's University, Belfast, 2013,

[15] Boussuge, F., Léon, J.-., Hahmann, S., "An analysis of the transformation requirements for digital mock-ups of structural assembly simulations," Civil-Comp Proceedings of 8th International Conference on Engineering Computational Technology, ECT 2012, Vol. 100, 2012.

[16] Mas, F., Menendez, J.L., Oliva, M., "IDMU as the collaborative engineering engine: Research experiences in airbus," International Conference on Engineering, Technology and Innovation: Engineering Responsible Innovation in Products and Services, ICE 2014, 2014.

[17] Riascos, R., Levy, L., Stjepandic, J., "Digital Mock-up," Concurrent Engineering in the 21st Century, edited by Stjepandic J. Wognum N. Verhagen WJC., Springer International Publishing, Switzerland, 2015, pp. 355-388. doi: 10.1007/978-3-319-13776-6_13

[18] Shapiro, V., and Srinivasan, V., "Report from a 2013 ASME panel on geometric interoperability for advanced manufacturing," CAD Computer Aided Design, Vol. 47, 2014, pp. A1-A2. doi: 10.1016/j.cad.2013.10.003

[19] Zubairi, M.S., Robinson, T.T., Armstrong, C.G., "A sensitivity approach for eliminating clashes from computer aided design model assemblies," Journal of Computing and Information Science in Engineering, Vol. 14, No. 3, 2014. 
doi: $10.1115 / 1.4027345$

[20] Franciosa, P., Gerbino, S., and Patalano, S., "Simulation of variational compliant assemblies with shape errors based on morphing mesh approach," International Journal of Advanced Manufacturing Technology, Vol. 53, No. 1-4, 2011, pp. 47-61. doi: 10.1007/s00170-010-2839-4

[21] Xu, S., and Keyser, J., "Geometric computation and optimization on tolerance dimensioning," CAD Computer Aided Design, Vol. 46, No. 1, 2014, pp. 129-137.

doi: 10.1016/j.cad.2013.08.025

[22] Ghali, M., Tlija, M., Aifaoui, N., "A CAD method for tolerance allocation considering manufacturing difficulty based on FMECA tool," International Journal of Advanced Manufacturing Technology, Vol. 91, No. 5-8, 2017, pp. 2435-2446.

doi: $10.1007 / \mathrm{s} 00170-016-9961-\mathrm{x}$

[23] Geis, A., Husung, S., Oberänder, A., "Use of vectorial tolerances for direct representation and analysis in CADsystems," Procedia CIRP, Vol. 27, 2015, pp. 230-240. doi: 10.1016/j.procir.2015.04.071

[24] Chan, Q., Zhenyu, L., Xiang, P., "Realistic Geometry Based Feature Modeling of Complex Part and Its Application in Assembly Quality Analysis," Journal of Computing and Information Science in Engineering, Vol. 15, No. 4, 2015.doi: 10.1115/1.4030462

[25] Liu, Y.S., and Gao, S.M., "An approach for generating variational geometry of a pattern of holes with composite positional tolerances," Proceedings of the ASME Design Engineering Technical Conference, Vol. 2, 2002, pp. 3-13.

doi: 10.1115/DETC2002/DAC-34033

[26] Tlija, Mehdi \& Korbi, Anis \& Louhichi, Borhen \& Abdelmajid, Benamara. (2019). A Novel Model for the tolerancing of non-rigid part assemblies in CAD. Journal of Computing and Information Science in Engineering. doi: 10.1115/1.4043528.

[27] Korbi, A., Tlija, M., Louhichi, B. et al. CAD/tolerancing integration: a new approach for tolerance analysis of non-rigid parts assemblies. Int J Adv Manuf Technol 98, 2003-2013 (2018).

doi: 10.1007/s00170-018-2347-5 
[28] Qin, Y., Lu, W., Liu, X., "Description logic-based automatic generation of geometric tolerance zones," International Journal of Advanced Manufacturing Technology, Vol. 79, No. 5-8, 2015, pp. 1221-1237. doi: $10.1007 / \mathrm{s} 00170-015-6839-2$

[29] Litwa, F., Gottwald, M., Bohn, M., "Automated point-based tolerance analysis model creation for sheet metal parts," Procedia CIRP, Vol. 27, 2015, pp. 65-70. doi: 10.1016/j.procir.2015.04.045

[30] Friel, I., Butterfield, J., Marzano, A., "Intelligent DMU Creation: Toleranced Part Modelling to Enhance the Digital Environment," Procedia CIRP, Vol. 60, 2017, pp. 92-97. doi: 10.1016/j.procir.2017.01.038

[31] CATIA, "Computer Aided Three-Dimensional Interactive Application," Dassault Systèmes, Ver.5 , France, 2013.

[32] "Python", Python Software Foundation, Ver. 2.7, Delaware, United States, 2010.

[33] Wan Din, W.I., "Sensitivity analysis in tolerance allocation," Queen's University, Belfast, Vol. 10, No. 2, 2013, pp. 139-151.

[34] Robinson, T., Friel, I., Armstrong, C.G., "Computer-aided design model parameterisation to derive knowledge useful for manufacturing design decisions," Proceedings of the Institution of Mechanical Engineers, Part B: Journal of Engineering Manufacture, Vol. 232, No. 4, 2017, pp. 621-628. doi: $10.1177 / 0954405417708218$

[35] Price, M.A., Robinson, T.T., Soban, D., "Maintaining design intent for aircraft manufacture," CIRP Annals Manufacturing Technology, Vol. 62, No. 1, 2013, pp. 99-102. doi: 10.1016/j.cirp.2013.03.124

[36] Bentley, J.L., "Multidimensional Binary Search Trees Used for Associative Searching," Communications of the ACM, Vol. 18, No. 9, 1975, pp. 509-517.

doi: $10.1145 / 361002.361007$

[37] Greenspan, M., and Yurick, M., "Approximate k-d tree search for efficient ICP," Proceedings of International Conference on 3-D Digital Imaging and Modeling, 3DIM, Vol. 2003-January, 2003, pp. 442-448. doi: 10.1109/IM.2003.1240280 
[38] Mao, J., Cao, Y.L., Liu, S.Q., "Manufacturing environment-oriented robust tolerance optimization method," International Journal of Advanced Manufacturing Technology, Vol. 41, No. 1-2, 2009, pp. 57-65. doi: 10.1007/s00170-008-1460-2

[39] ISO, T.2., "ISO 2768-1:1989, General tolerances - Part 1: Tolerances for linear and angular dimensions without individual tolerance indications," American National Standards Institute (ANSI), 2007. 